
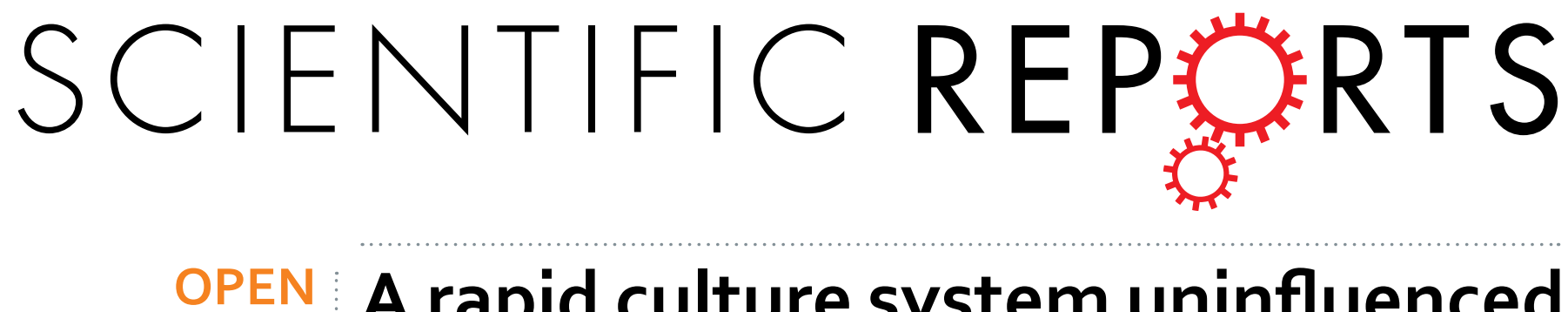

\title{
A rapid culture system uninfluenced by an inoculum effect increases reliability and convenience for drug susceptibility testing of Mycobacterium tuberculosis
}

Received: 21 June 2017

Accepted: 8 May 2018

Published online: 05 June 2018
Yong-Gyun Jung ${ }^{1,2,3}$, Hyejin $\mathrm{Kim}^{4}$, Sangyeop Lee ${ }^{5}$, Suyeoun Kim${ }^{5}$, EunJi Jo5, Eun-Geun Kim Jungil Choi ${ }^{6}{ }^{6}$, Hyun Jung Kim ${ }^{5}$, Jungheon Yoo ${ }^{5}$, Hye-Jeong Lee ${ }^{4}$, Haeun Kim $\mathbb{1}^{4}{ }^{4}$, Hyunju Jung ${ }^{4}$, Sungweon Ryoo ${ }^{9}$ \& Sunghoon Kwon ${ }^{5,6,7,8}$

The Disc Agarose Channel (DAC) system utilizes microfluidics and imaging technologies and is fully automated and capable of tracking single cell growth to produce Mycobacterium tuberculosis (MTB) drug susceptibility testing (DST) results within 3 7 days. In particular, this system can be easily used to perform DSTs without the fastidious preparation of the inoculum of MTB cells. Inoculum effect is one of the major problems that causes DST errors. The DAC system was not influenced by the inoculum effect and produced reliable DST results. In this system, the minimum inhibitory concentration (MIC) values of the first-line drugs were consistent regardless of inoculum sizes ranging from $\sim 10^{3}$ to $\sim 10^{8} \mathrm{CFU} / \mathrm{mL}$. The consistent MIC results enabled us to determine the critical concentrations for 12 anti-tuberculosis drugs. Based on the determined critical concentrations, further DSTs were performed with 254 MTB clinical isolates without measuring an inoculum size. There were high agreement rates $(96.3 \%)$ between the DAC system and the absolute concentration method using Löwenstein-Jensen medium. According to these results, the DAC system is the first DST system that is not affected by the inoculum effect. It can thus increase reliability and convenience for DST of MTB. We expect that this system will be a potential substitute for conventional DST systems.

Tuberculosis (TB) remains a major global health concern; it killed 1.5 million people in $2014^{1}$. The key to stopping TB transmission is rapid diagnosis and correct treatment with regimens based on drug susceptibility tests. To cope with and detect the emergence of resistant TB, much effort has been devoted to improving diagnostic tools based on culture and molecular techniques.

Molecular DSTs based on the detection of genotypic mutations are advantageous for the rapid screening of drug resistant $\mathrm{TB}$, but there are critical gaps because the correlation of genotypic mutations with drug resistance are not fully understood and because the molecular DSTs have been applied only in some drug-resistant cases ${ }^{2,3}$. Additionally, these DSTs cannot detect all mutations involved in resistance, because the commercialized tests (line probe assays and Xpert MTB/RIF) only cover certain genes and regions (e.g. a limited resistance-determining

${ }^{1}$ Interdisciplinary Program of Biomodulation, Myongji University, Yongin, Gyeonggi-do, 17058, Republic of Korea. ${ }^{2}$ Myongji Bioefficiency Research Centre, Myongji University, Yongin, Gyeonggi-do, 17058, Republic of Korea. ${ }^{3}$ Center for Nutraceutical and Pharmaceutical Materials, Myongji University, Yongin, Gyeonggi-do, 17058, Republic of Korea. ${ }^{4}$ Korean Institute of Tuberculosis, Osong, Cheongju, Chung Buk, 28158, Republic of Korea. ${ }^{5}$ QuantaMatrix Inc., Seoul National University Hospital CMI, Jongno-gu, Seoul, 03082, Republic of Korea. ${ }^{6}$ Department of Electrical Engineering and Computer Science, Seoul National University, Seoul, 08826, Republic of Korea. ${ }^{7}$ Institutes of Entrepreneurial BioConvergence, Seoul National University, Seoul, 08826, Republic of Korea. ${ }^{8}$ Seoul National University Hospital Biomedical Research Institute, Seoul National University Hospital, Seoul, 03080, Republic of Korea. ${ }^{9} \mathrm{Clinical}$ Research Centre, Masan National Tuberculosis Hospital, Changwon, Korea. Yong-Gyun Jung and Hyejin Kim contributed equally to this work. Correspondence and requests for materials should be addressed to S.R. (email: viweon@gmail.com) or S.Kw. (email: skwon@snu.ac.kr) 
region (RRDR) of the $r p o B$ gene) ${ }^{4}$. Culture-based DSTs, called "phenotypic DSTs", analyze viable cells grown either in broth or on solid medium and detect phenotypic resistance. The phenotype DST methods are widely accepted as a gold standard by the World Health Organization (WHO), but have not been widely performed owing to their disadvantages: (1) they are time-consuming, (2) they present a risk of potential infection and (3) the results of phenotype DST methods are not fully reproducible ${ }^{2,5}$.

MGIT 960 (Becton Dickinson, MD, USA) is a liquid-culture system that can shorten the DST running time from the 4 6 weeks of the Löwenstein-Jensen (L-J) method to 13 days. Although MGIT 960 offers faster DST results than solid methods, it still has many barriers to overcome, as do another conventional methods ${ }^{6,7}$. First, one of the main barriers is preparing the proper cell number at the inoculum stage. The culture-based DST methods require a strict standardized inoculum size to produce reproducible DST results. However, in the MTB DST, it is not easy to evenly homogenize clumps of waxy-coated MTB cells; the clumps possibly cause inadequate cell numbers at the inoculum stage, leading to the inoculum effect (IE) during DST. IE is one of the major problems that cause DST errors, leading to reduced drug activity from the increased bacterial cell density ${ }^{8-10}$. Consequently, IE can increase the MICs of anti-TB drugs, resulting in irreproducible DST results. Secondly, the procedures involved in the inoculum preparation and culturing are always a concern from the standpoint of biosafety because the MTB cell suspension is serially diluted to adjust the correct cell density and this step could generate MTB aerosols, resulting in increased risk of laboratory TB transmission.

To date, there have been various approaches to shorten the turn-around time of DSTs, but none have attempted to overcome the inoculum effect even though it influences the reproducibility of DST results. Using microfluidics and imaging technologies, we designed and developed the Disc Agarose Channel system, which is fully automated and capable of tracking single cell growth to produce MTB DST results within 3 7 days ${ }^{11}$.

In the present study, we observed that this system is not influenced by inoculum size. To verify this finding, we evaluated the consistency of MIC values with various inoculum sizes in the range of $\sim 10^{3}$ to $\sim 10^{8} \mathrm{CFU} / \mathrm{mL}$, as well as determined the critical concentration and validated the DAC system without measuring inoculum size on clinical isolates for clinical application. These results showed that the DAC system is not affected by inoculum size, and we observed strong agreement (96.3\% overall agreement for the 12 tested anti-TB drugs) between the DAC system and the L-J method (reference method).

\section{Materials and Methods}

Strains. The M. tuberculosis H37Rv ATCC 27294 and total 484 clinical MTB strains containing Pansusceptible, multidrug resistant (MDR) and extensively drug resistant (XDR) isolates were obtained from the Korean Institute of Tuberculosis (KIT). All procedures for MTB cultures and DSTs were performed using a biological safety cabinet (BSC) inside the KIT's biosafety level 3 (BSL3) laboratory. Drug susceptibility results of all clinical isolates in this study were previously determined by the absolute concentration method using L-J medium prepared in-house $\mathrm{e}^{12,13}$.

Analysis of activities of anti-TB drugs with various inoculum concentrations. For the inoculum effect study, all isolates were freshly sub-cultured on L-J medium before being used. The colonies were vortexed in a tube containing a few drops of PBS and glass beads, to break up the large clumps. The bacterial cells were inoculated as a set $5 \times 10^{3} \sim 5 \times 10^{8} \mathrm{CFU} / \mathrm{mL}$ including the standard inoculum of bacterial concentration $\left(\sim 5 \times 10^{5}\right.$ $\mathrm{CFU} / \mathrm{mL})$. To create high bacterial density $\left(\sim 10^{8} \mathrm{CFU} / \mathrm{mL}\right)$, the dense suspension was centrifuged for 10 minutes at $3,000 \mathrm{~g}$ and the pellet was resuspended in sterile PBS to achieve $\sim 10^{8} \mathrm{CFU} / \mathrm{mL}$. Then, this bacterial suspension was serially diluted. The cell numbers were counted by the bacterial CFU method on Middlebrook 7H11 agar (BD $\mathrm{BBL}, \mathrm{MD}$, USA) plates with the easySpiral plater system (Interscience, Saint Nom la Breteche, France). To estimate the MIC values for the 4 first-line drugs, five concentrations were tested according to two-fold serial dilutions. MTB H37Rv and two clinical isolates were prepared as described above. The MICs for each drug were estimated with various inoculum sizes, ranging from $\sim 10^{3}$ to $\sim 10^{8} \mathrm{CFU} / \mathrm{mL}$. To compare the appropriate inoculum size among three DST tests, L-J DST and MGIT 960 DST were both performed by the standard methods according to a previously described study ${ }^{14,15}$. The DSTs of three different methods with H37Rv were performed with differing inoculum sizes, ranging from $\sim 10^{4}$ to $\sim 10^{7} \mathrm{CFU} / \mathrm{mL}^{16}$. The critical concentrations (CCs) of the DAC system were adopted as the breakpoints of the BACTEC 460 TB and MGIT 960 systems, based on the Middlebrook 7H9 (BD $\mathrm{BBL}, \mathrm{MD}$, USA) broth, because it was reported that the CCs were dependent on the medium ${ }^{17}$, and the DAC system was also based on the Middlebrook 7H9 broth. The CCs of the DAC system were $0.1 \mu \mathrm{g} / \mathrm{mL}$ for INH, $1.0 \mu \mathrm{g} / \mathrm{mL}$ for RIF, $1.0 \mu \mathrm{g} / \mathrm{mL}$ for STR, and $5.0 \mu \mathrm{g} / \mathrm{mL}$ for EMB.

Broth microdilution test. The broth microdilution (BMD) test was used as a gold standard recommended by the Clinical and Laboratory Standards Institute (CLSI) ${ }^{18}$. The extended spectrum beta-lactamase (ESBL)-negative E. coli ATCC 25922 strain was purchased from ATCC and clinical the ESBL-positive E. coli was obtained at $\mathrm{SNUH}$ as described previously ${ }^{19}$. For the BMD test, the cefepime solutions were prepared from the stock solution. A $100 \mu \mathrm{L}$ volume of the antimicrobial agent at the appropriate concentration, which was determined by the CLSI recommendation ${ }^{18}$, was pipetted into the bottom of 96 -microwell plates (Falcon/BD Biosciences, CA, USA). Ten microliters of bacterial stock solution was inoculated into the wells at final concentrations of $10^{5} \sim 10^{7} \mathrm{CFU} / \mathrm{mL}$. After $16 \sim 20 \mathrm{~h}$ of incubation at $37^{\circ} \mathrm{C}$, the MIC values of the microdilution wells were read as the concentration at which there was $\geq 99 \%$ reduction in growth compared to the control, by unaided visual inspection.

Drug susceptibility test. The DST method of the DAC system was previously described ${ }^{11}$. We mixed $300 \mu \mathrm{L}$ of the MTB cell suspension with $900 \mu \mathrm{L}$ of $0.5 \%$ agarose at $37^{\circ} \mathrm{C}$ by vortexing. Subsequently, $40 \mu \mathrm{L}$ of $0.375 \%$ agarose mixture with MTB cell suspension was loaded into the inlet of a DAC chip. The agarose was then 
A

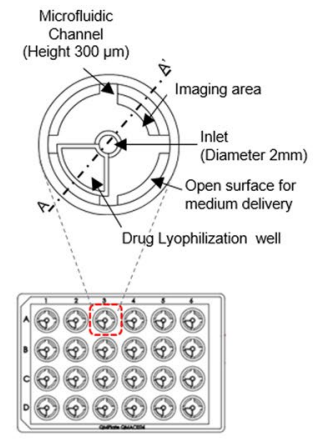

B
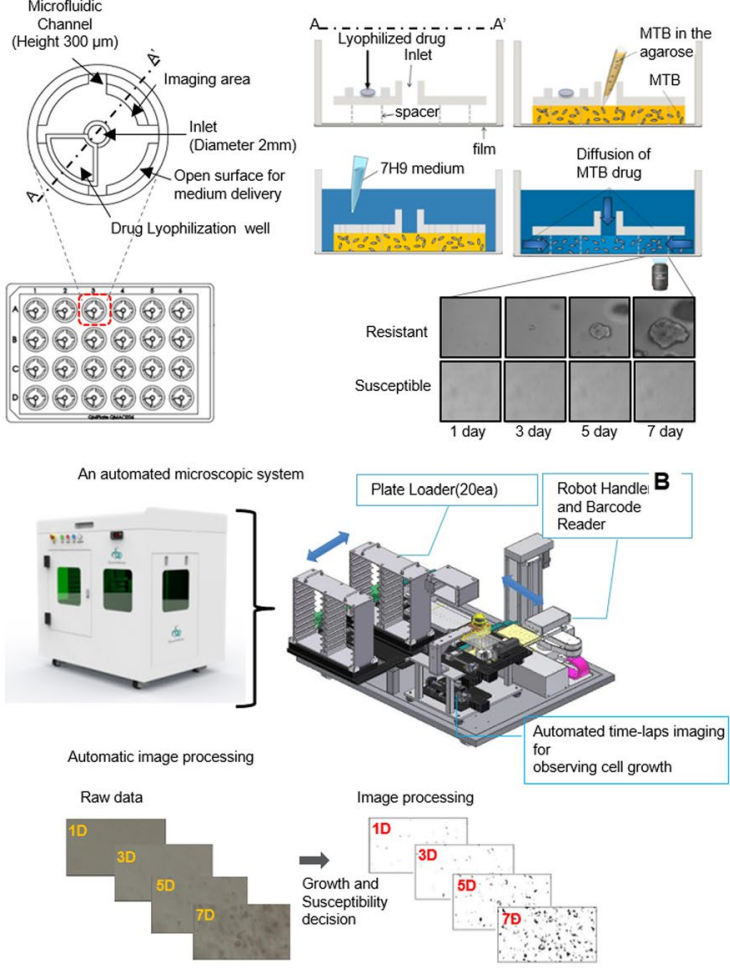

c

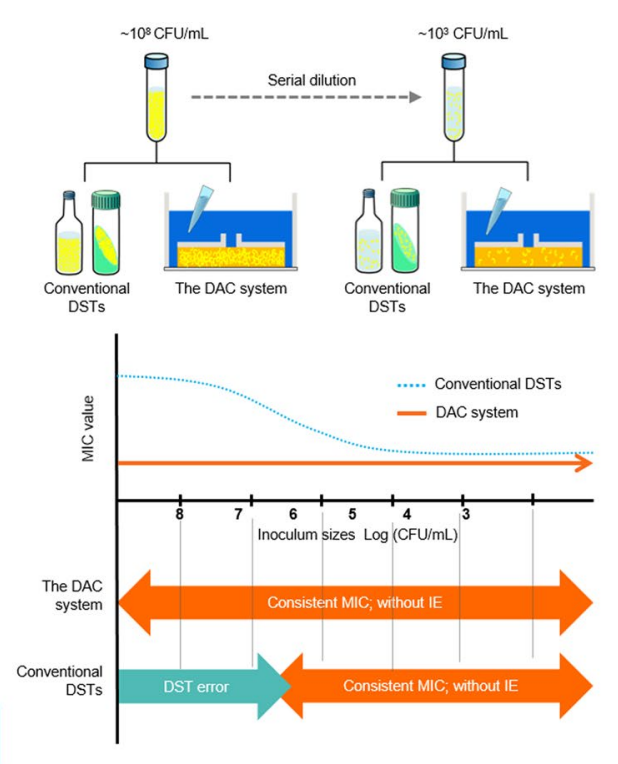

Figure 1. The fully automated DAC system performs DST-based MTB imaging and has no inoculum effect, leading to reliable DST results. (A) Schematic of the DAC chip containing 12 anti-TB lyophilized drugs. The detailed methods for DAC DST are as described in the Materials and Methods. (B) In an automated microscopic system, the DAC chip was loaded and unloaded on the reader and one area at the edge of the agarose was automatically imaged every other day using the time-lapse method. Then, growth images were automatically processed. (C) Advantages of the DAC system compared with conventional solid and liquid DST systems for MTB: there are inoculum effects over $\sim 10^{6} \mathrm{CFU} / \mathrm{mL}$ in the conventional DST systems that can cause DST errors, but no inoculum effect is observed in the DAC system, leading to consistent MIC values regardless of inoculum sizes from $\sim 10^{3} \mathrm{CFU} / \mathrm{mL}$ to $\sim 10^{8} \mathrm{CFU} / \mathrm{mL}$.

allowed to solidify at room temperature for 1 minute. Each TB drug was lyophilized and added into each well. The lyophilized drug resolved immediately after a $0.5 \mathrm{~mL}$ addition of the $7 \mathrm{H} 9$ broth containing $10 \%$ OADC; the proper concentration of each drug was adjusted. The resolved drug in the culture medium was then allowed to diffuse into the agarose. After this process, the DAC chip was then sealed by an air-permeable film for safety and prevention of contamination and incubated in a temperature-controlled culture chamber at $37^{\circ} \mathrm{C}$ for 7 days. One area at the edge of the agarose was automatically imaged with a $20 \times$ lens on an inverted microscope every other day using the time-lapse method. Growth images were then automatically processed (Fig. 1).

Determination of critical concentrations in the DAC system. Based on the critical concentrations of the BACTEC 460 TB and MGIT 960 systems for each drug recommended by the WHO policy guidelines on DST of second-line anti-TB drugs in 2008, five concentrations were chosen according to two-fold serial dilutions. Strains were chosen from 89 well-characterized susceptible groups obtained from new cases and 141 resistant groups obtained during drug treatment from pulmonary tuberculosis patients (Supplementary Table S1). After graphing the curve of MIC distribution, the CCs for each drug were determined at the concentration where the cumulative percentage difference between susceptible and resistant strains mostly showed the greatest percentage difference as previously described ${ }^{13}$.

Validation of the DAC system. The DAC system established CCs was validated with a total of 254 clinical MTB strains including pan-susceptible strains and resistant strains tested by the L-J method. Each drug was lyophilized in the DAC chip according to its critical concentration. To ensure the consistency of lyophilized drugs, the reference strain MTB H37Rv ATCC 27294 and the clinical isolate KIT87190 strain were used as internal controls for each test of DST ${ }^{20}$. DSTs of the DAC system were performed without measuring the MTB inoculum size and the DST results of the DAC system were compared with those of the L-J method. For a blinded assessment of the outcomes, results from the DAC system were determined automatically using an imaging processing program without knowledge of the results from the reference method.

Resolution of discrepancy between the DAC system and the L-J method. The discrepant results between the DAC system and the L-J method were confirmed by DNA sequence analysis ${ }^{21,22}$. We performed DNA 
sequencing by using the target regions involved in the following genes as previous described ${ }^{22} ; r p o B$ (RRDR) for rifampin and rifabutin, $e m b B$ for ethambutol, $r r s$ and $r p s L$ for streptomycin, $g y r A$ for quinolones, and $r r s$, eis and tly $A$ for kanamycin, amikacin and capreomycin, respectively. Target regions for each gene are $r p o B=759807-$ 763325, embB $=4246517-4249810$, rrs $=1471846-1473382$, $r p s L=781560-781934$, gyr $A=7302-9818$, eis $=2714124-2715477$, and $t l y A=1917940-1918746$ of the H37Rv genome sequence (GenBank accession number NC_000962.3) 22 .

Antimicrobial agents and drug lyophilization in the DAC chip. All drugs were purchased from Sigma-Aldrich (St. Louis, MO, USA) including the first line drugs (isoniazid (INH), rifampin (RIF), streptomycin (STR), ethambutol (EMB), and rifabutin (RFB)) and the second-line drugs (amikacin (AMI), capreomycin (CAP), kanamycin (KAN), levofloxacin (LEV), moxifloxacin (MOXI), ofloxacin (OFL), and para-aminosalicylic acid (PAS)). Stock solutions of each antibiotic were prepared in accordance with the manufacturer's instructions and working solutions were prepared fresh from the stock solution. For drug lyophilization, all drugs in the DAC chip were freeze-dried under a vacuum, and the freezing process was carried out using a freeze dryer (Ilshin Biobase Co., LTD., Korea). After freeze-drying, the DAC chip was packaged in an aluminum foil vacuum-sealed pouch and stored at $4^{\circ} \mathrm{C}$ before use. The 12 lyophilized anti-TB drugs in the DAC chip were validated by MIC determination using the clinical isolate KIT87190 strain. Quality control ranges of MIC value for each drug were determined from three different batches twice a week for 5 weeks, resulting in 30 replicates in total (Supplementary Table S2) ${ }^{23}$.

\section{Results}

Activities of anti-TB drugs in the DAC system are not affected by inoculum size. Because the DAC system is a new method based on microfluidics and imaging technologies, the optimal bacterial density at the inoculum step needed to be determined by comparison with the MIC values from the conventional DST methods. First, we estimated the MICs for the first-line drugs (INH, RIF, STR and EMB) with the various inoculum sizes in the range of $\sim 10^{4}$ to $\sim 10^{7} \mathrm{CFU} / \mathrm{mL}$. The MIC values from the different inoculum sizes of MTB H37Rv, the standard laboratory strain, were determined and compared within 7 days. Surprisingly, consistent MICs were observed with essential agreement regardless of the inoculum size: The MIC values were $0.025 \mu \mathrm{g} / \mathrm{mL}$ and $0.05 \mu \mathrm{g} /$ $\mathrm{mL}$ for INH, $0.5 \mu \mathrm{g} / \mathrm{mL}$ and $1.0 \mu \mathrm{g} / \mathrm{mL}$ for RIF, $0.5 \mu \mathrm{g} / \mathrm{mL}$ and $1.0 \mu \mathrm{g} / \mathrm{mL}$ for STR, and $2.5 \mu \mathrm{g} / \mathrm{mL}$ and $5.0 \mu \mathrm{g} / \mathrm{mL}$ for EMB with inoculum sizes of $\sim 10^{4}$ to $\sim 10^{7} \mathrm{CFU} / \mathrm{mL}$, respectively (Fig. $2 \mathrm{~A}$ ).

To compare the DAC systems with the other conventional DST systems on the point of the inoculum effect, the DSTs of three different methods with $\mathrm{H} 37 \mathrm{Rv}$ were performed with various inoculum sizes from $\sim 10^{4}$ to $\sim 10^{7}$ $\mathrm{CFU} / \mathrm{mL}$. The DST results of the DAC system with H37Rv were all "susceptible (S)" regardless of the inoculum size. In contrast, the DST results of either the L-J method or MGIT 960 were "resistant (R)" or "system error" when the inoculum size was higher than $\sim 10^{6} \mathrm{CFU} / \mathrm{mL}$ (Fig. 2B). This result indicated that the DAC system was not sensitive to the inoculum effect, in contrast to the other conventional DST methods.

For further verification, we examined four clinical isolates, two pan-susceptible strains and two XDR MTB strains. In the DAC system, we did not observe any inoculum effect from MIC determinations of the clinical strains with various inoculum sizes from $\sim 10^{3}$ to $\sim 10^{8} \mathrm{CFU} / \mathrm{mL}$ (Fig. $2 \mathrm{C}$ ). The MIC values were determined with essential agreement regardless of inoculum size, with the exception of STR against two susceptible strains. The MIC values of STR were from 0.5 to $2.0 \mu \mathrm{g} / \mathrm{mL}$, but the highest value did not exceed $2.0 \mu \mathrm{g} / \mathrm{mL}$, which was the critical concentration of the BACTEC 460 TB system ${ }^{24,25}$.

For verification with extended clinical strains and random inoculum sizes, 110 clinical strains containing 31 pan-susceptible and 79 MDR MTB determined by the L-J method were tested. The DST results of the first-line drugs were determined and compared to those of the L-J method. The breakpoints of the BACTEC systems were employed as in Fig. 2B. The inoculums of all strains were randomly prepared without measuring cell density by four researchers. Then, 3 weeks afterward, they were counted by the bacterial CFU method on $7 \mathrm{H} 11$ agar plates. The highest inoculum size was $1.1 \times 10^{8} \mathrm{CFU} / \mathrm{mL}$, and the lowest was $1.4 \times 10^{5} \mathrm{CFU} / \mathrm{mL}$ (Fig. $3 \mathrm{~A}$ ) according to the CFU counting results. In spite of various inoculum sizes with $\sim 1,000$-fold ranges, there were high agreement rates (overall 95.2\% agreement) compared with the DST results of the L-J method (Fig. 3B).

With such consistent MIC data, the DAC system could minimize the inoculum effect that causes limitations in the in vitro drug susceptibility test ${ }^{26,27}$. To explain this phenomenon, the physical characteristics of the DAC system were considered. The enclosed environmental conditions of the agarose matrix may contribute to the minimization of the inoculum effect. To verify this hypothesis, the ESBL-negative E. coli ATCC 25922 and a clinical ESBL-positive E. coli strain were tested with inoculum concentrations of $5 \times 10^{5}$ to $5 \times 10^{7} \mathrm{CFU} / \mathrm{mL}$ and $0.015 \sim 128 \mu \mathrm{g} / \mathrm{mL}$ of cefepime (a beta-lactam antibiotic) in both the DAC system and the conventional BMD method. Interestingly, antimicrobial susceptibility tests against both ESBL-positive and -negative strains showed an inoculum effect for the BMD method, but not for the DAC system. The IE was more serious in the case of the ESBL-positive strain in the BMD method (Fig. 3C). This effect implies that in the BMD method, metabolites or proteins (beta-lactamase) produced by the ESBL-positive strain easily bind and inactivate cefepime, whereas in the DAC system, they could be trapped in the agarose, and cannot bind and inactivate the antibiotic, even at $\sim 5 \times 10^{7} \mathrm{CFU} / \mathrm{mL}$.

Determination of the critical concentration of anti-TB drugs in the DAC system. The CCs of five of the first-line and seven of the second-line anti-TB drugs in the DAC system were determined with 230 clinical isolates whose DST results were already well characterized by both the absolute L-J method and DNA sequence analysis. The inocula of all clinical strains were randomly prepared without measuring cell density.

The MIC values for the first-line drugs (INH, RIF, STR, EMB, and RFB) and the second-line drugs (AMI, CAP, KAN, LEV, MOXI, OFL, and PAS) were determined within 7 days. After determining the MIC of each drug, the $\mathrm{CCs}$ for each drug were established at the concentration where the cumulative percentage difference between 
A

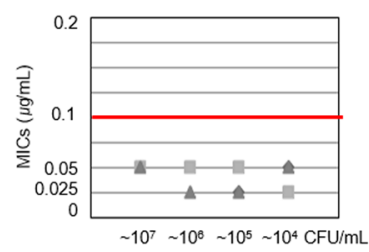

B

\begin{tabular}{|c|c|c|c|c|}
\hline \multirow{2}{*}{$\begin{array}{l}\text { DST } \\
\text { for INH }\end{array}$} & \multicolumn{4}{|c|}{ Inoculum sizes (CFU/mL) } \\
\hline & $\sim 10^{7}$ & $\sim 10^{\circ}$ & $\sim 10^{5}$ & $\sim 10^{4}$ \\
\hline L-J & $R$ & $\mathrm{R}$ & $\mathrm{s}$ & $\mathrm{s}$ \\
\hline MGIT & error & error & s & $\mathrm{s}$ \\
\hline DAC & $s$ & $\mathrm{~s}$ & $\mathrm{~s}$ & $\mathrm{~s}$ \\
\hline
\end{tabular}

RIF

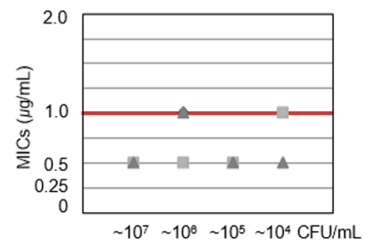

RIF

\begin{tabular}{ccccc}
\hline \multirow{2}{*}{$\begin{array}{c}\text { DST } \\
\text { for RIF }\end{array}$} & \multicolumn{4}{c}{ Inoculum sizes } \\
\cline { 2 - 5 } & $\sim 10^{7}$ & $\sim 10^{6}$ & $\sim 10^{5}$ & $\sim 10^{4}$ \\
\hline L-J & $\mathrm{R}$ & $\mathrm{S}$ & $\mathrm{S}$ & $\mathrm{S}$ \\
MGIT & error & error & $\mathrm{S}$ & $\mathrm{S}$ \\
DAC & $\mathrm{S}$ & $\mathrm{S}$ & $\mathrm{S}$ & $\mathrm{S}$ \\
\hline
\end{tabular}

STR

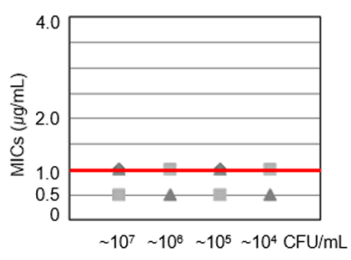

STR

\begin{tabular}{ccccc}
\hline \multirow{2}{*}{$\begin{array}{c}\text { DST } \\
\text { for STR }\end{array}$} & \multicolumn{3}{c}{ Inoculum sizes (CFU/mL) } \\
\cline { 2 - 5 } & $\sim 10^{7}$ & $\sim 10^{\circ}$ & $\sim 10^{5}$ & $\sim 10^{4}$ \\
\hline L-J & $\mathrm{R}$ & $\mathrm{R}$ & $\mathrm{S}$ & $\mathrm{S}$ \\
MGIT & error & error & $\mathrm{S}$ & $\mathrm{S}$ \\
DAC & $\mathrm{S}$ & $\mathrm{S}$ & $\mathrm{S}$ & $\mathrm{S}$ \\
\hline
\end{tabular}

Resistant strains
C
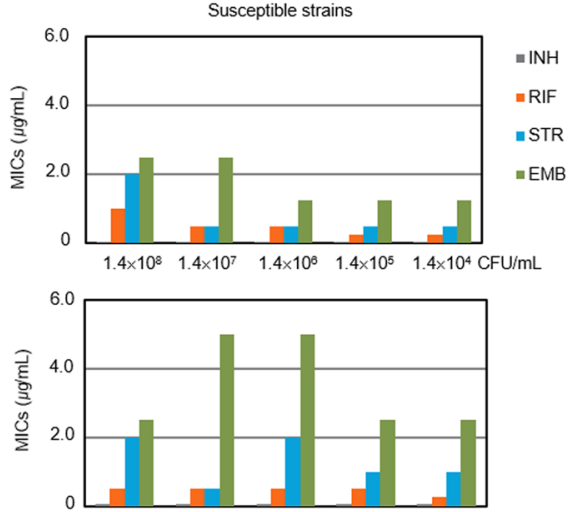
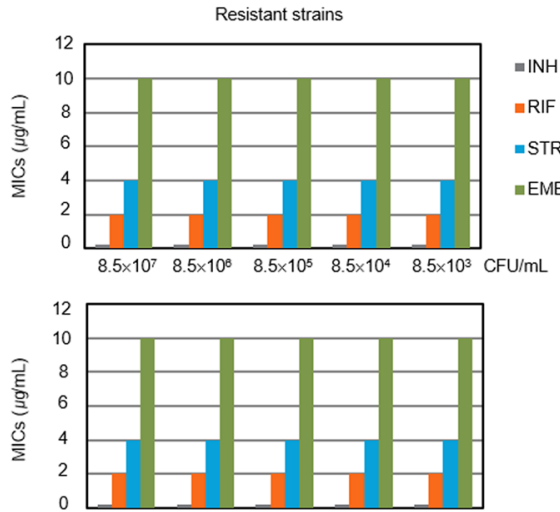

Figure 2. Activities of anti-TB TB drugs in the DAC system are not affected by inoculum size. (A) The MIC values for the first-line drugs according to various inoculum sizes. MTB H37Rv ATCC 27294 cells from $\sim 10^{4}$ to $\sim 10^{7} \mathrm{CFU} / \mathrm{mL}$ were inoculated in the DAC system and the MIC values were determined. The spots (circle, triangle and square) of each drug indicate the MICs values from three independently repeated experiments. The tested concentrations for each drug were a two-fold dilution scale. The breakpoints of the BACTEC 460 TB and MGIT 960 systems based on the Middlebrook 7H9 broth were adopted for the DAC system; $0.1 \mu \mathrm{g} / \mathrm{mL}$ for INH, $1.0 \mu \mathrm{g} / \mathrm{mL}$ for RIF, $1.0 \mu \mathrm{g} / \mathrm{mL}$ for STR, and $5.0 \mu \mathrm{g} / \mathrm{mL}$ for EMB. The red horizontal line indicates the breakpoints for each drug. All MIC values were determined under the breakpoints. (B) The comparison of an inoculum effect for the first-line drugs against $\mathrm{H} 37 \mathrm{Rv}$ between the DAC system and two routine methods, the L-J method (solid) and MGIT 960 method (liquid). The various inoculum sizes from $\sim 10^{4}$ to $\sim 10^{7} \mathrm{CFU} / \mathrm{mL}$ were tested. The DST results were represented as resistant $(\mathrm{R})$ or susceptible $(\mathrm{S})$. The DST results were consistently "S" regardless of the various inoculum sizes in the DAC system, whereas they were changed from "S" to "R" or "Error" over $5 \times 10^{6} \mathrm{CFU} / \mathrm{mL}$ in the two routine methods. (C) The MICs values from clinical isolates in the various inoculum sizes. The MIC values from two pan-susceptible and two resistant strains were estimated for the first-line drugs. The inoculum sizes were from $\sim 10^{3} \mathrm{CFU} / \mathrm{mL}$ to $\sim 10^{8} \mathrm{CFU} / \mathrm{mL}$ for two drug susceptible strains and two drug resistant strains. There was no inoculum effect with the clinical isolates in the DAC system.

susceptible and resistant strains mostly showed the greatest percentage difference except for STR and RFB (Fig. 4). The determined CCs were $0.1 \mu \mathrm{g} / \mathrm{mL}$ for INH, $1.0 \mu \mathrm{g} / \mathrm{mL}$ for RIF, $2.0 \mu \mathrm{g} / \mathrm{mL}$ for STR, $5.0 \mu \mathrm{g} / \mathrm{mL}$ for EMB, $2.0 \mu \mathrm{g} /$ $\mathrm{mL}$ for AMI, $2.5 \mu \mathrm{g} / \mathrm{mL}$ for CAP, $2.5 \mu \mathrm{g} / \mathrm{mL}$ for KAN, $1.5 \mu \mathrm{g} / \mathrm{mL}$ for LEV, $0.5 \mu \mathrm{g} / \mathrm{mL}$ for MOXI, $2.0 \mu \mathrm{g} / \mathrm{mL}$ for OFL, $0.5 \mu \mathrm{g} / \mathrm{mL}$ for RFB, and $4.0 \mu \mathrm{g} / \mathrm{mL}$ for PAS.

For the CC of STR, there was the greatest percentage difference at $1.0 \mu \mathrm{g} / \mathrm{mL}$. However, it was reported that the MIC range of STR against the MTB H37Rv and H37Ra by the BACTEC system were 0.094 0.75 and 0.38 1.5 $\mu$ g/ $\mathrm{mL}$, respectively ${ }^{28}$, and the CC of STR was $2.0 \mu \mathrm{g} / \mathrm{mL}$ in the BACTEC system ${ }^{24,25}$. In addition, the MIC values of STR against the susceptible MTB strains isolated from new patients before drug treatment showed $1.0 \mathrm{or} 2.0 \mu \mathrm{g} /$ $\mathrm{mL}$ repeatedly in the DAC system. For these reasons, we determined that the CC of STR was $2.0 \mu \mathrm{g} / \mathrm{mL}$ in the DAC system. In the case of RFB, $0.125 \mu \mathrm{g} / \mathrm{mL}$ showed the greatest percentage difference, but $0.5 \mu \mathrm{g} / \mathrm{mL}$ was determined as the critical concentration according to the BACTEC system because the CC determination is dependent on the medium and both the DAC system the BACTEC system used the same $7 \mathrm{H} 9$ broth. There was no significant difference in the agreement rate between $0.125 \mu \mathrm{g} / \mathrm{mL}(96.9 \%)$ and $0.5 \mu \mathrm{g} / \mathrm{mL}(94.8 \%)$.

Validation of the automated DAC system. In the previous work, the DAC system was fully automated with an image-processing program for rapid DST. The DSTs of the first-line drugs were performed with $\mathrm{H} 37 \mathrm{Rv}$ 
A

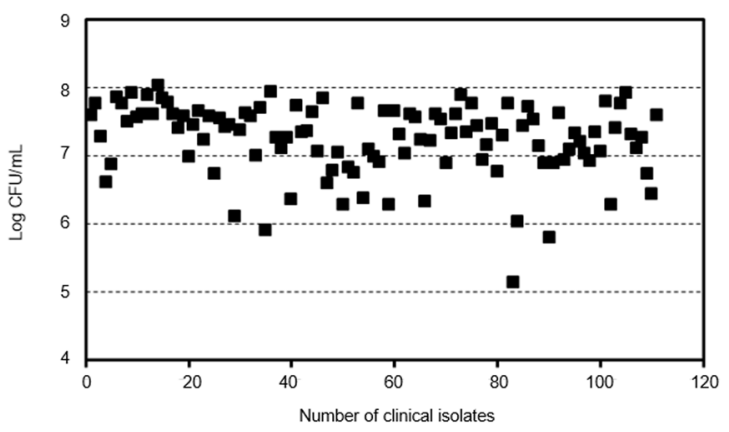

B

\begin{tabular}{|c|c|c|c|c|c|c|}
\hline \multirow[t]{2}{*}{$\begin{array}{l}\text { Drug } \\
(\mu g / m \ell)\end{array}$} & \multirow[t]{2}{*}{$\begin{array}{l}\text { No. } \\
\text { tested }\end{array}$} & \multicolumn{2}{|c|}{$\begin{array}{l}\text { Agreement } \\
\text { (DAC / L-J) }\end{array}$} & \multicolumn{2}{|c|}{$\begin{array}{l}\text { Discrepancy } \\
\text { (DAC/L-J) }\end{array}$} & \multirow[t]{2}{*}{$\begin{array}{c}\text { Overall } \\
\text { Agreement rate }\end{array}$} \\
\hline & & $R / R^{a}$ & $\mathrm{~S} / \mathrm{S}^{\circ}$ & $R / S$ & $S / R$ & \\
\hline $\mathrm{INH}(0.1)$ & 110 & 79 & 31 & 0 & 0 & $100 \%(110 / 110)$ \\
\hline $\operatorname{RIF}(1.0)$ & 110 & 79 & 31 & 0 & 0 & $100 \%(110 / 110)$ \\
\hline $\operatorname{STR}(2.0)$ & 110 & 65 & 37 & 8 & 0 & $92.7 \%(102 / 110)$ \\
\hline $\operatorname{EMB}(5.0)$ & 110 & 66 & 37 & 2 & 5 & $93.6 \%(103 / 110)$ \\
\hline
\end{tabular}

C
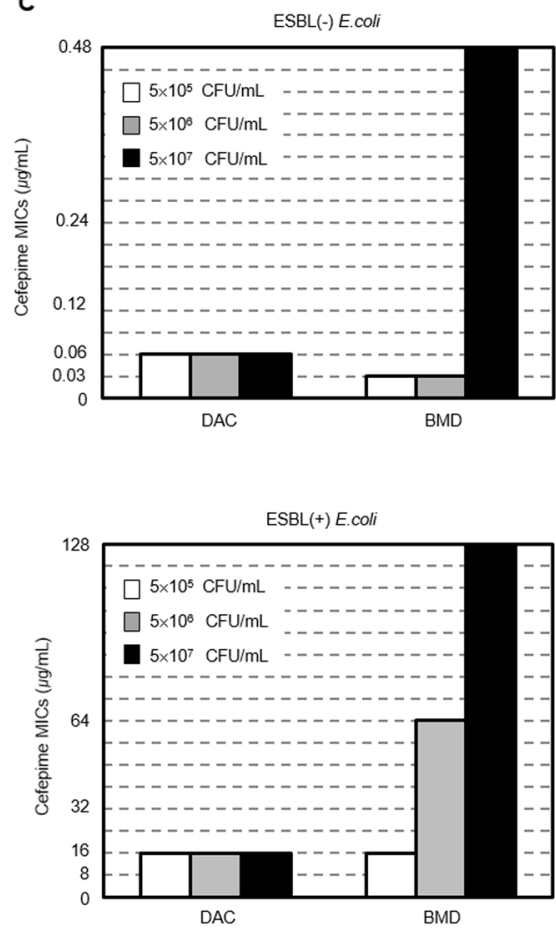

Figure 3. DSTs with the randomly prepared inocula of clinical isolates. (A) The distribution of random inoculum sizes prepared without measuring cell density. Four researchers randomly prepared $110 \mathrm{clinical}$ isolates, and the bacterial CFUs were counted afterward. The highest inoculum size was $1.1 \times 10^{8} \mathrm{CFU} / \mathrm{mL}$ and the lowest was $1.4 \times 10^{5} \mathrm{CFU} / \mathrm{mL}$. (B) DST results with the randomly prepared inocula with clinical isolates. The DSTs for the first-line drugs were performed with the randomly prepared inocula in the DAC system, and the DST results were compared with those of the L-J method. There were high agreement rates between the two systems. (C) More severe inoculum effect in the ESBL-positive strain. Antibiotic-inactivating enzymes in the culture medium caused a more severe inoculum effect with respect to the use of cefepime according to the inoculum size. In the broth microdilution method, the inoculum effect in the ESBL-negative E. coli ATCC 25922 strain was over $10^{7} \mathrm{CFU} / \mathrm{mL}$, and the inoculum effect of the ESBL-positive E. coli strain was over $10^{6} \mathrm{CFU} / \mathrm{mL}$. However, in the DAC system, there was no inoculum effect with either the ESBL-negative or ESBL-positive strains from $10^{5} \mathrm{CFU} / \mathrm{mL}$ to $10^{7} \mathrm{CFU} / \mathrm{mL}$.

and $\sim 30$ clinical isolates ${ }^{11}$. In this work, to create a more convenient system, each drug was lyophilized in the DAC chip, and the activities of lyophilized drugs showed consistency within quality control ranges of 12 anti-TB drugs at $4{ }^{\circ} \mathrm{C}$ for 6 months (Table 1 and Supplementary Table S2). Additionally, we obtained reproducible results for each drug, between tests done during different weeks by three different operators (Supplementary Table S3). To validate the CCs of this system in a clinical setting, DSTs of the first-line and second-line drugs were performed with 254 clinical isolates including susceptible and resistant MTB strains categorized by the L-J method. The inocula of all clinical strains were randomly prepared without measuring cell density. The DST results were compared with those of the reference method (the L-J method). INH and RIF showed very high agreements (100\% and $99.6 \%$, respectively) for both susceptible and resistant strains. The agreement rates for the other anti-TB drugs were also high, in the range of $91.3 \sim 99.2 \%$. The overall agreement rate for all drugs was $96.3 \%$ (Table 2). Sixty-seven strains showed discrepant results between the DAC and the reference method. To resolve any discrepancy between-tests, we analyzed some discrepant results using DNA sequence analysis ${ }^{21,22}$. The comparison of results after their resolution is shown in Table 3. The DNA sequencing results of the target genes showed a better correlation when using the DAC system (62.1\%) than when using the L-J method (37.9\%). Although the L-J method has been considered the gold standard for phenotypic DST for MTB, they may show falsely low MICs, causing the resistant strain to be evaluated as susceptible to the tested drug, compared with the molecular DST results of whole-genome sequencing ${ }^{29}$, suggesting that the DAC system provides more accurate DST results than the conventional method.

Safety of the DAC system. In the MTB DST, safety is one of the most important issues. MTB cells can often infect lab researchers during DST procedures through MTB aerosol generation from serial dilution, or accidental leakage of MTB cells from test tubes or wells in a plate ${ }^{30}$. We had already demonstrated that the DAC system did not need serial dilution for preparation of the inoculum. The DAC system features extra safety devices, such as a sealing film and a locking lid. The MTB cells in the DAC system were embedded in the solidified agarose matrix, so that the suspended MTB cells could be minimized in the broth medium (Fig. 5A). Figure 5B shows the 

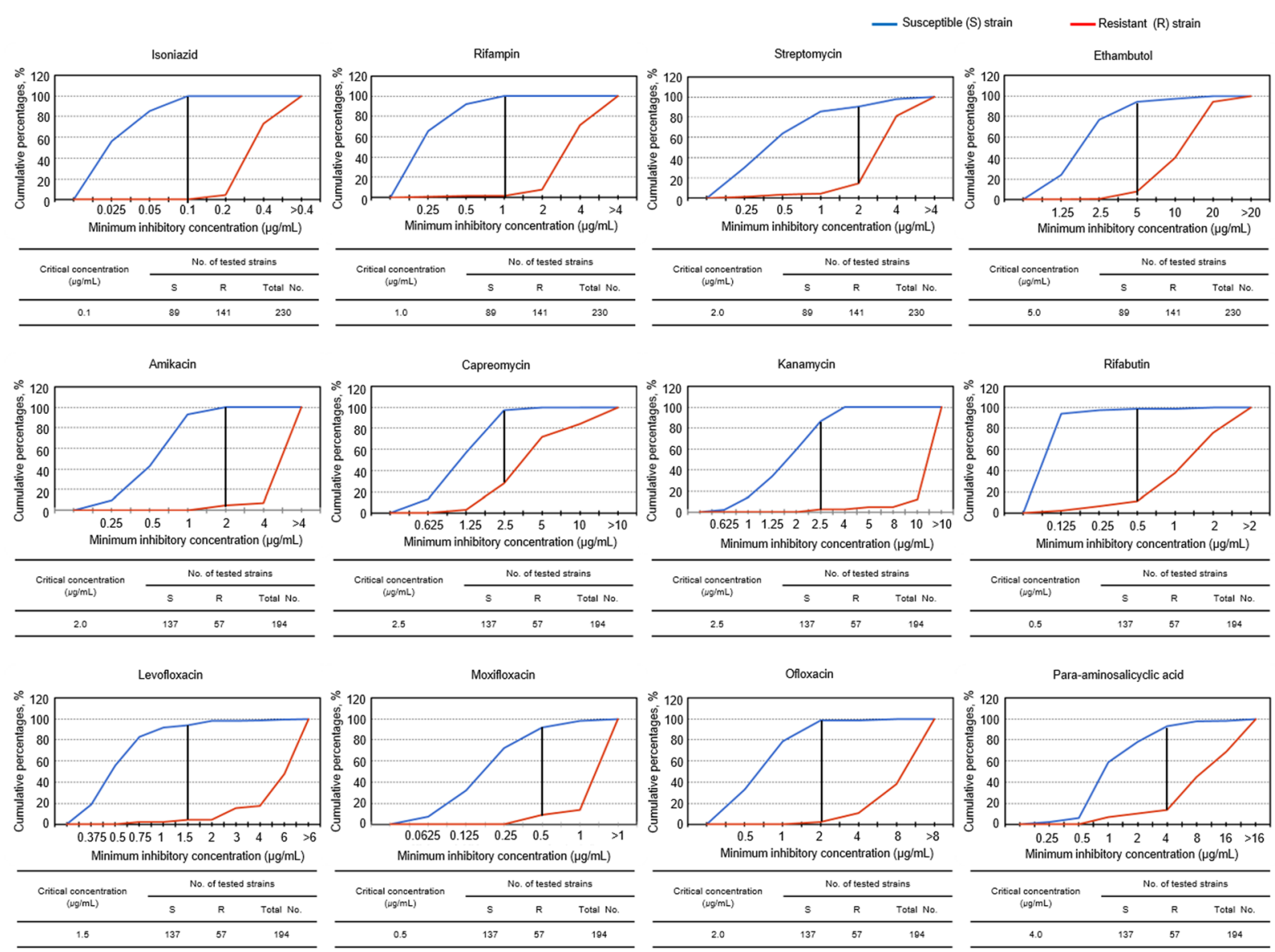

Figure 4. Determination of critical concentrations in the DAC system. Cumulative percentage plots of susceptible and resistant strains against respective MIC $(\mu \mathrm{g} / \mathrm{mL})$ values for each drug in the DAC system were obtained. Critical concentrations for 12 anti-TB drugs were determined mainly on the concentrations showing the greatest percentage difference. The numbers of the tested susceptible and resistant strains for each drug were shown in the table. The black number on the right side of the vertical line on the critical concentration shows the greatest percentage difference between susceptible strains and resistant strains, except STR and RFB.

comparison of MTB cell counts between the DAC and the liquid culture systems in the broth medium. H37Rv was inoculated with $4 \times 10^{5} \mathrm{CFU} / \mathrm{mL}$ into both the broth of the liquid culture system and the agarose matrix of the DAC system. At 1, 3, 5, and 7 days after inoculation, the supernatants from both devices were taken, and MTB cells were counted according to the CFU method. In the liquid culture system, MTB cells were observed in the broth after 1 day; the cell count increased from $4 \times 10^{5} \mathrm{CFU} / \mathrm{mL}$ to $1.4 \times 10^{7} \mathrm{CFU} / \mathrm{mL}$. However, in the DAC system, MTB cells were detected in the broth only after 5 days, at $1.5 \times 10^{2} \mathrm{CFU} / \mathrm{mL}$; this count had slightly increased after 7 days, to $6.2 \times 10^{3} \mathrm{CFU} / \mathrm{mL}$. Taken together, the DAC system can efficiently prevent the accidental leakage of MTB cells during the DSTs to reduce TB infection risk.

\section{Discussion}

There are some reasons that the MTB DSTs have not been performed easily and widely: (1) safety issues (2) irreproducible DST results and (3) a long turn-around time (4 to 6 weeks) $)^{2,31}$. The DAC system shows that it is possible to overcome these barriers. The MTB cells in the DAC system are embedded in the solidified agarose matrix, so that the suspended MTB cells can be minimized in the broth medium, to reduce the risk of contact from liquid medium leakage. The inoculum size is one of the main factors underlying these barriers, and inappropriate inoculum cell concentrations lead to DST errors ${ }^{6,8,17}$. The DAC system can produce faster and more reproducible DST results regardless of the inoculum size (in the range of $10^{3} \sim 10^{8}$ cell $/ \mathrm{mL}$ ) within 7 days. There was no inoculum effect in this system, and it was not necessary to measure McFarland turbidity values of the MTB cells to prepare the proper inoculum size.

In previous studies, the duration for which drug concentration remained above the MIC (\% T $>$ MICs) was measured in in vivo animal models, and there was no significant difference in $\% \mathrm{~T}>$ MIC required for drug efficacy, regardless of inoculum size or ESBL production status; on the other hand, the MIC values of in vitro tests were increased for high inoculum size ${ }^{32}$. This pattern suggested that the IE could be an artifact caused by the limitations of in vitro DST methods ${ }^{26,27}$. Based on this hypothesis, the DAC system could mimic in vivo DSTs more than the other conventional in vitro DST systems. 


\begin{tabular}{|c|c|c|c|c|c|c|c|c|c|c|c|c|c|}
\hline & Drug & INH & RIF & STR & EMB & AMI & CAP & KAN & LEV & MOXI & OFL & PAS & RFB \\
\hline & $\mathrm{CC}(\mu \mathrm{g} / \mathrm{mL})$ & 0.1 & 1.0 & 2.0 & 5.0 & 2.0 & 2.5 & 2.5 & 1.5 & 0.5 & 2.0 & 4.0 & 0.5 \\
\hline Month & $\begin{array}{l}\text { Quality } \\
\text { control range }\end{array}$ & $0.025 \sim 0.05$ & $0.125 \sim 0.5$ & $0.25 \sim 1.0$ & $1.25 \sim 2.5$ & $0.25 \sim 1.0$ & $0.625 \sim 2.5$ & $0.625 \sim 2.5$ & 0.375 & $0.0625 \sim 0.25$ & $0.5 \sim 1.0$ & $1.0 \sim 4.0$ & $0.125 \sim 0.25$ \\
\hline \multirow{8}{*}{1} & \multirow{2}{*}{ Fresh (1 3) } & 0.05 & 0.25 & 0.5 & 5.0 & 1.0 & 0.625 & 1.25 & 0.375 & 0.125 & 1.0 & 1.0 & 0.125 \\
\hline & & (S) & (S) & (S) & (S) & (S) & (S) & (S) & (S) & (S) & (S) & (S) & (S) \\
\hline & \multirow{2}{*}{1} & 0.05 & 0.25 & 0.5 & 2.5 & 1.0 & 2.5 & 2.5 & 0.375 & 0.125 & 1.0 & 1.0 & 0.125 \\
\hline & & $(\mathrm{S})$ & $(\mathrm{S})$ & (S) & $(\mathrm{S})$ & (S) & (S) & $(\mathrm{S})$ & (S) & (S) & (S) & (S) & (S) \\
\hline & \multirow{2}{*}{2} & 0.025 & 0.25 & 0.25 & 2.5 & 1.0 & 1.25 & 2.5 & 0.75 & 0.125 & 1.0 & 1.0 & 0.125 \\
\hline & & (S) & (S) & (S) & (S) & (S) & (S) & (S) & (S) & (S) & (S) & (S) & (S) \\
\hline & \multirow{2}{*}{3} & 0.025 & 0.25 & 0.5 & 5.0 & 1.0 & 2.5 & 2.5 & 0.375 & 0.25 & 1.0 & 1.0 & 0.125 \\
\hline & & $(\mathrm{S})$ & $(\mathrm{S})$ & (S) & $(\mathrm{S})$ & $(\mathrm{S})$ & (S) & (S) & (S) & (S) & (S) & (S) & (S) \\
\hline \multirow{8}{*}{2} & \multirow{2}{*}{ Fresh (1 3) } & 0.05 & 0.25 & 0.25 & 2.5 & 0.25 & 0.625 & 1.25 & 0.375 & 0.125 & 0.5 & 1.0 & 0.125 \\
\hline & & $(\mathrm{S})$ & $(\mathrm{S})$ & $(\mathrm{S})$ & $(\mathrm{S})$ & (S) & (S) & $(\mathrm{S})$ & (S) & (S) & (S) & (S) & (S) \\
\hline & \multirow{2}{*}{1} & 0.05 & 0.25 & 0.25 & 2.5 & 0.5 & 1.25 & 1.25 & 0.375 & 0.125 & 0.5 & 1.0 & 0.125 \\
\hline & & (S) & $(\mathrm{S})$ & $(\mathrm{S})$ & $(\mathrm{S})$ & (S) & (S) & (S) & (S) & (S) & (S) & (S) & (S) \\
\hline & \multirow{2}{*}{2} & 0.025 & 0.25 & 0.25 & 2.5 & 0.5 & 0.625 & 1.25 & 0.375 & 0.125 & 0.5 & 1.0 & 0.125 \\
\hline & & (S) & (S) & (S) & (S) & (S) & (S) & (S) & (S) & (S) & (S) & (S) & (S) \\
\hline & \multirow{2}{*}{3} & 0.05 & 0.25 & 0.25 & 2.5 & 0.5 & 0.625 & 1.25 & 0.375 & 0.125 & 0.5 & 1.0 & 0.125 \\
\hline & & (S) & (S) & (S) & (S) & (S) & (S) & (S) & (S) & (S) & (S) & (S) & (S) \\
\hline \multirow{10}{*}{3} & \multirow{2}{*}{ Fresh (1) } & 0.025 & 0.5 & 1.0 & 2.5 & 0.5 & 1.25 & 2.5 & 0.375 & 0.125 & 0.5 & 1.0 & 0.125 \\
\hline & & (S) & (S) & (S) & $(\mathrm{S})$ & (S) & $(\mathrm{S})$ & $(\mathrm{S})$ & (S) & (S) & (S) & (S) & (S) \\
\hline & \multirow{2}{*}{ Fresh $(2,3)$} & 0.025 & 0.25 & 0.25 & 2.5 & 0.5 & 1.25 & 2.5 & 0.375 & 0.125 & 0.5 & 1.0 & 0.125 \\
\hline & & (S) & (S) & (S) & (S) & (S) & (S) & (S) & (S) & (S) & (S) & (S) & (S) \\
\hline & \multirow{2}{*}{1} & 0.05 & 0.5 & 1.0 & 5.0 & 0.5 & 1.25 & 1.25 & 0.375 & 0.0625 & 0.5 & 1.0 & 0.125 \\
\hline & & (S) & (S) & (S) & $(\mathrm{S})$ & (S) & (S) & $(\mathrm{S})$ & (S) & (S) & (S) & (S) & (S) \\
\hline & \multirow{2}{*}{2} & 0.025 & 0.25 & 0.25 & 2.5 & 0.25 & 0.625 & 1.25 & 0.375 & 0.0625 & 0.5 & 1.0 & 0.125 \\
\hline & & (S) & $(\mathrm{S})$ & $(\mathrm{S})$ & (S) & (S) & (S) & (S) & (S) & (S) & (S) & (S) & (S) \\
\hline & \multirow{2}{*}{3} & 0.025 & 0.5 & 0.5 & 2.5 & 0.5 & 1.25 & 2.5 & 0.375 & 0.125 & 0.5 & 1.0 & 0.125 \\
\hline & & (S) & (S) & (S) & (S) & (S) & $(\mathrm{S})$ & (S) & (S) & (S) & (S) & (S) & (S) \\
\hline \multirow{8}{*}{4} & \multirow{2}{*}{ Fresh (1 3) } & 0.025 & 0.5 & 1.0 & 5.0 & 1.0 & 2.5 & 2.5 & 0.75 & 0.25 & 1.0 & 4.0 & 0.125 \\
\hline & & (S) & (S) & (S) & $(\mathrm{S})$ & (S) & (S) & $(\mathrm{S})$ & (S) & (S) & (S) & (S) & (S) \\
\hline & \multirow{2}{*}{1} & 0.05 & 0.5 & 1.0 & 5.0 & 1.0 & 2.5 & 2.5 & 0.375 & 0.25 & 1.0 & 2.0 & 0.125 \\
\hline & & (S) & (S) & (S) & (S) & (S) & (S) & (S) & (S) & (S) & (S) & (S) & (S) \\
\hline & \multirow{2}{*}{2} & 0.05 & 0.25 & 0.5 & 2.5 & 1.0 & 2.5 & 2.5 & 0.75 & 0.25 & 1.0 & 1.0 & 0.125 \\
\hline & & $(\mathrm{S})$ & $(\mathrm{S})$ & (S) & (S) & (S) & (S) & (S) & (S) & (S) & (S) & $(\mathrm{S})$ & (S) \\
\hline & 3 & 0.05 & 0.25 & 0.5 & 2.5 & 1.0 & 2.5 & 2.5 & 0.375 & 0.25 & 0.5 & 1.0 & 0.125 \\
\hline & 3 & $(\mathrm{~S})$ & $(\mathrm{S})$ & (S) & $(\mathrm{S})$ & (S) & (S) & $(\mathrm{S})$ & (S) & (S) & (S) & (S) & (S) \\
\hline & Frech $(1 \sim 3)$ & 0.05 & 0.25 & 0.5 & 2.5 & 1.0 & 1.25 & 2.5 & 0.375 & 0.25 & 1.0 & 2.0 & 0.125 \\
\hline & Fresh $(1 \sim 3)$ & (S) & (S) & (S) & (S) & (S) & (S) & (S) & (S) & (S) & (S) & (S) & (S) \\
\hline & 1 & 0.05 & 0.25 & 0.25 & 2.5 & 0.5 & 1.25 & 1.25 & 0.375 & 0.125 & 0.5 & \begin{tabular}{|l|}
1.0 \\
\end{tabular} & 0.125 \\
\hline 6 & 1 & (S) & (S) & (S) & (S) & (S) & (S) & (S) & (S) & (S) & (S) & (S) & (S) \\
\hline 6 & 2 & 0.05 & 0.25 & 0.25 & 2.5 & 0.5 & 2.5 & 1.25 & 0.375 & 0.125 & 0.5 & 1.0 & 0.125 \\
\hline & 2 & (S) & (S) & (S) & (S) & (S) & (S) & (S) & (S) & (S) & (S) & (S) & (S) \\
\hline & 3 & 0.025 & 0.25 & 0.25 & 2.5 & 0.5 & 1.25 & 2.5 & 0.375 & 0.125 & 0.5 & 2.0 & 0.125 \\
\hline & 3 & (S) & (S) & (S) & (S) & (S) & (S) & (S) & (S) & (S) & (S) & (S) & (S) \\
\hline
\end{tabular}

Table 1. Validation of the 12 lyophilized anti-TB drugs. The 12 lyophilized anti-TB drugs in the DAC chip were validated by MIC determination using the clinical isolate KIT87190 strain. Activities of lyophilized drugs showed consistency at $4^{\circ} \mathrm{C}$ for 6 months with quality control ranges of each drug. As a control, fresh drugs (Fresh) were prepared and the MIC values were determined. The tests were repeated three times.

There are two possibilities why the inoculum effect did not occur in the DAC system: (1) the absolute amount of MTB cells is below the range in which the inoculum effect appears. In the DAC system, $10^{3} \sim 10^{8}$ cells $/ \mathrm{mL}$ bacterial cell suspension is diluted with agarose at a 1:3 dilution ratio, and the agarose-cell solution contains $2.5 \times 10^{2} \sim 2.5 \times 10^{7}$ cells $/ \mathrm{mL}$ of bacterial cells. The $40 \mu \mathrm{L}$ mixture was loaded, and then $0.5 \mathrm{~mL}$ of liquid medium was added to the well. The final cell number for DST becomes $5.0 \times 10^{0} \sim 5.0 \times 10^{5} \mathrm{cells} / \mathrm{mL}$. The inoculum size of $5.0 \times 10^{5}$ cells $/ \mathrm{mL}$ is hardly known to have an inoculum effect according to CLSI guidelines ${ }^{18}$. (2) The enclosed environmental conditions in the agarose matrix may contribute to the minimization of the inoculum effect. In the DAC system, the MTB cells are immobilized by agarose, and their metabolites or proteins that inhibit antibiotic 


\begin{tabular}{|c|c|c|c|c|c|c|c|c|c|c|}
\hline \multirow[b]{2}{*}{$\operatorname{Drug}(\mu \mathrm{g} / \mathrm{mL})$} & \multirow{2}{*}{$\begin{array}{l}\text { No. } \\
\text { tested }\end{array}$} & \multicolumn{2}{|c|}{$\begin{array}{l}\text { Agreement } \\
\text { (DAC/L-J) }\end{array}$} & \multicolumn{2}{|c|}{$\begin{array}{l}\text { Discrepancy } \\
\text { (DAC/L-J) }\end{array}$} & \multirow{2}{*}{$\begin{array}{l}\text { Overall Agreement } \\
\text { Rate }\end{array}$} & \multirow{2}{*}{$\begin{array}{l}\text { Sensitivity } \\
(\%)\end{array}$} & \multirow{2}{*}{$\begin{array}{l}\text { Specificity } \\
(\%)\end{array}$} & \multirow{2}{*}{$\begin{array}{l}\text { PPV } \\
\text { (\%) }\end{array}$} & \multirow{2}{*}{$\begin{array}{l}\text { NPV } \\
(\%)\end{array}$} \\
\hline & & $\mathbf{R} / \mathbf{R}^{\mathbf{a}}$ & $\mathbf{S} / \mathbf{S}^{\mathbf{b}}$ & $\mathrm{R} / \mathrm{S}$ & $\mathbf{S} / \mathbf{R}$ & & & & & \\
\hline $\mathrm{INH}(0.1)$ & 254 & 127 & 127 & 0 & 0 & $100 \%(254 / 254)$ & 100 & 100 & 100 & 100 \\
\hline $\operatorname{RIF}(1.0)$ & 254 & 124 & 129 & 0 & 1 & $99.6 \%(253 / 254)$ & 99.2 & 100 & 100 & 99.2 \\
\hline STR (2.0) & 254 & 65 & 171 & 15 & 3 & $92.9 \%(236 / 254)$ & 95.6 & 91.9 & 81.3 & 98.3 \\
\hline EMB (5.0) & 254 & 115 & 133 & 5 & 1 & $97.6 \%(248 / 254)$ & 99.1 & 96.4 & 95.8 & 99.3 \\
\hline AMI (2.0) & 254 & 79 & 168 & 4 & 3 & $97.2 \%(247 / 254)$ & 96.3 & 97.7 & 95.2 & 98.2 \\
\hline CAP (2.5) & 254 & 63 & 174 & 13 & 4 & $93.3 \%(237 / 254)$ & 94.0 & 93.0 & 82.9 & 97.8 \\
\hline KAN (2.5) & 254 & 89 & 155 & 9 & 1 & $96.1 \%(244 / 254)$ & 98.9 & 94.5 & 90.8 & 99.4 \\
\hline LEV (1.5) & 254 & 86 & 160 & 2 & 6 & $96.9 \%(246 / 254)$ & 93.5 & 98.8 & 97.7 & 96.4 \\
\hline MOXI (0.5) & 254 & 81 & 158 & 15 & 0 & $94.1 \%(239 / 254)$ & 100 & 91.3 & 84.4 & 100 \\
\hline OFL (2.0) & 254 & 96 & 156 & 0 & 2 & $99.2 \%(252 / 254)$ & 98.0 & 100 & 100 & 98.7 \\
\hline PAS (4.0) & 254 & 61 & 171 & 13 & 9 & $91.3 \%(232 / 254)$ & 87.1 & 92.9 & 82.4 & 95 \\
\hline RFB (0.5) & 254 & 91 & 156 & 4 & 3 & $97.2 \%(247 / 254)$ & 96.8 & 97.5 & 95.8 & 98.1 \\
\hline
\end{tabular}

Table 2. Comparison of validation results by the L-J method and the DAC system. We tested 254 MTB clinical isolates without measuring the inoculum sizes and all drugs were lyophilized in the DAC chip. Some of discrepant results were further analyzed via DNA sequencing (materials and methods). ${ }^{a} \mathrm{R}$ : resistant, ${ }^{\mathrm{b}} \mathrm{S}$ : susceptible.

\begin{tabular}{|l|l|l|c|c|}
\hline Drug & $\begin{array}{l}\text { No. Discrepancy } \\
\text { (DAC/L-J) }\end{array}$ & $\begin{array}{l}\text { No. tested by } \\
\text { Sequencing }\end{array}$ & $\begin{array}{l}\text { Agreement } \\
\text { (DAC/Sequencing) }\end{array}$ & $\begin{array}{l}\text { Agreement } \\
\text { (L-J/Sequencing) }\end{array}$ \\
\hline RIF & 1 & 1 & $1 / 1(100 \%)$ & $0 / 1(0 \%)$ \\
\hline STR & 18 & 11 & $7 / 11(63.6 \%)$ & $4 / 11(36.4 \%)$ \\
\hline EMB & 6 & 4 & $2 / 4(50.0 \%)$ & $2 / 4(50.0 \%)$ \\
\hline AMI & 7 & 7 & $4 / 7(57.1 \%)$ & $3 / 7(42.9 \%)$ \\
\hline CAP & 17 & 13 & $9 / 13(69.2 \%)$ & $4 / 13(30.8 \%)$ \\
\hline KAN & 10 & 3 & $1 / 3(33.3 \%)$ & $2 / 3(66.7 \%)$ \\
\hline LEV & 8 & 7 & $5 / 7(71.4 \%)$ & $2 / 7(28.6 \%)$ \\
\hline MOXI & 15 & 12 & $4 / 12(33.3 \%)$ & $8 / 12(66.7 \%)$ \\
\hline OFL & 2 & 2 & $2 / 2(100 \%)$ & $0 / 2(0 \%)$ \\
\hline RFB & 7 & 7 & $3 / 7(42.9 \%)$ & $4 / 7(57.1 \%)$ \\
\hline
\end{tabular}

Table 3. Comparison of discrepant results after resolution by DNA sequence analysis. We analyzed 67 discrepant DST results by DNA sequencing for the target genes (Materials and Methods).

activity could also be trapped in the agarose matrix, thus preventing easy binding and inactivation of antibiotics in the broth. In Fig. 3C, the result implies that beta-lactamase in the DAC system could be trapped in the agarose, and cannot bind and inactivate the antibiotic, even at high inoculum size.

The resolution results of the DAC system showed a better correlation with DNA sequencing when compared with the conventional method, but the cases (MOXI, KAN, and RFB) showed lower correlation by resolution (Table 3). For MOXI, four isolates showing the "R" agreement in the DAC system $(4 / 12,33.3 \%)$ were detected to have mutations in gyrA. The other eight isolates (" $\mathrm{S}$ " result in the L-J method and " $\mathrm{R}$ " in the DAC system) had a mutation in gyrA (S95T) as well, but this mutation is not associated with resistance ${ }^{32}$. To clarify MOXI sequencing results, it is necessary to analyze all regions associated with fluoroquinolone resistance, such as efflux pumps, gyrB, and the other regions of gyrA, despite showing low frequcency ${ }^{33,34}$. For RFB, three isolates showing "R" agreement in the DAC system $(3 / 7,42.9 \%)$ were detected as having SNPs causing S531L and H526Y in the $r p o B$ region known as the resistant region. Among four discrepant isolates ("S" results in the DAC system), three were detected to have the same SNP (S531L), but one was observed to have an H516Y substitution in showing rare rifampicin or RFB susceptible isolates in the MGIT 960 DST $^{21}$. For KAN, among 10 discrepant isolates, three isolates were tested by sequencing the $r r s$, eis, and tlyA regions. There was no mutation in three isolates, whereas mutations were detected in the rrs (nucleotide A1401G) region in two of these strains, no mutations were found in the eis and tly $A$ regions. Further studies may be necessary to understand the genetic basis of these phenotypes.

There are systems for rapid DSTs such as the MGIT 960 and MODS systems. The MGIT 960 system can generally produce DST results in 9 to 13 days and is commercialized, but this system has a severe inoculum effect (Fig. 2B) and detects cell growth by an indirect method, e.g., measuring fluorescence rates depending on the amount of oxygen consumption. In addition, the DST results are easily spoiled by bacterial contamination. In the microscopic observation drug susceptibility assay (MODS) system, MTB growth is measured by cord formation (a direct measuring method), and DST results can be produced in 5 to 14 days. However, it has been reported that some MTB cells cannot form cords $\mathrm{s}^{35}$, and therefore, DST results are not reproducible. The MODS also poses a safety risk in the DST procedure. Therefore, the MODS system is not easily fully automated and has not yet been commercialized in the clinical area. The DAC system is fully automated and enables us to produce rapid and 
A

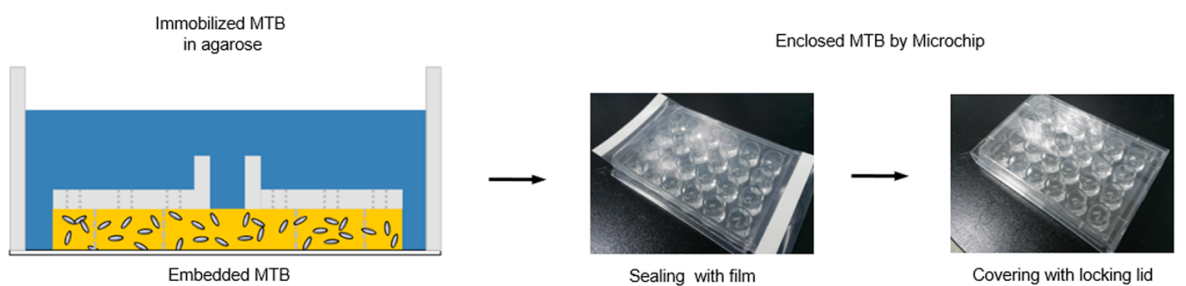

B
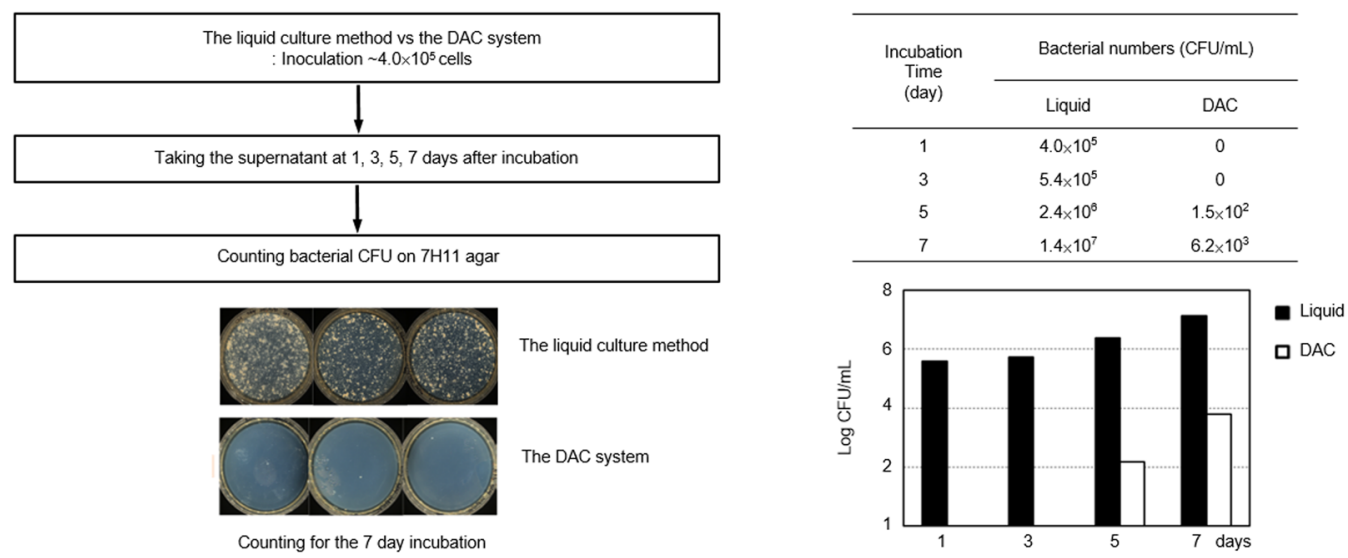

Figure 5. Safety of the DAC system. (A) The DAC system is safer for researchers as it blocks the leakage of MTB cells in three ways. (1) MTB cells in the DAC chip are immobilized in the solidified agarose matrix. (2) Each well in the chip is enclosed with sealing film and (3) the DAC chip is securely covered with a locking lid. (B) The comparison of MTB H37Rv cell numbers in the culture medium between the DAC chip and the liquid culture method after inoculation. MTB cells $\left(\sim 4.0 \times 10^{5} \mathrm{CFU} / \mathrm{mL}\right)$ were inoculated both in the liquid culture system and in the DAC system. To perform this safety test, a $40-\mu \mathrm{L}$ of the agarose-MTB mixture was loaded onto a DAC chip and $0.5 \mathrm{~mL}$ of the $7 \mathrm{H} 9$ medium was then added. After 1-, 3-, 5- and 7-day incubations, supernatants $(50 \mu \mathrm{L})$ from both systems were collected using a pipette: The cells were plated on $7 \mathrm{H} 11$ agar plates, and 3 weeks later of culture, the bacterial cells were counted using the bacterial CFU method. In the DAC system, bacterial cells were not detected in culture broth until 1 and 3 incubation days, and there were $\sim 1.5 \times 10^{2}$ and $\sim 6.2 \times 10^{3} \mathrm{MTB}$ cells on the 5th and 7th days, respectively. There were 1,000-fold fewer cells than in the liquid culture method.

accurate MIC results regardless of the inoculum size and provides a safer DST process in one week, demonstrating that this system can be a better substitute for conventional DST systems. Although the DAC system has great potential to be used in the clinic, there remains some work to do. PZA is one of the first-line drugs but was not included in the DAC system because the DST culture condition for PZA is different from the other drugs. The DAC system needs a protocol for the PZA test so that it can be included. This system was validated with more than 250 clinical samples, but additional validation processes are needed with more clinical strains from various clinical sites.

\section{References}

1. World Health Organization-Global Tuberculosis Report. http://www.who.int/tb/publications/global_report/en/index.html (2015).

2. Simons, S. O. \& van Soolingen, D. Drug susceptibiliy testing for optimizing tuberculosis treatment. Current Pharmaceutical Design. 17, 2863-2874 (2011)

3. Boehme, C. C. et al. Rapid molecular detection of tuberculosis and rifampin resistance. N Engl J Med. 363, 1005-1015 (2010).

4. Steingart, K. R. et al. Xpert ${ }^{\circledR}$ MTB/RIF assay for pulmonary tuberculosis and rifampicin resistance in adults. Cochrane Database Syst Rev. 31, CD009593 (2014)

5. Minion, J., Leung, E., Menzies, D. \& Pai, M. Microscopic-observation drug susceptibility and thin layer agar assays for the detection of drug resistant tuberculosis: a systematic review and meta-analysis. Lancet. 10, 688-698 (2010).

6. Kim, S. J. Drug-susceptibility testing in tuberculosis: methods and reliability of results. Eur Respir J. 25, 564-569 (2005).

7. Rodrigues, C. et al. Drug susceptibility testing of Mycobacterium tuberculosis against second-line drugs using the Bactec MGIT 960 system. Int J Tuberc Lung Dis. 12, 1449-1455 (2008).

8. Mitchison, D. A. Drug resistance in tuberculosis. Eur Respir J. 25, 376-379 (2005).

9. Brook, I. Inoculum effect. Rev Infect Dis. 11, 361-368 (1989).

10. Woods, G. L. et al. Susceptibility testing of Mycobacteria, Nocardiae, and other Aerobic Actinomycetes; Approved Standard-Second edition. Document M24-A2. Clinical and Laboratory Standards Institute, Wayne, PA (2011).

11. Choi, J. I. et al. Rapid drug susceptibility test of Mycobacterium tuberculosis using microscopic time-lapse imaging in an agarose matrix. Appl Microbiol Biotechnol. 100, 2355-2365 (2016).

12. Canetti, G. et al. Mycobacteria: laboratory methods for testing drug sensitivity and resistance. Bull world Health Organ. 29, 565-578 (1963).

13. Kam, M. A. et al. Determination of critical concentrations of second-line anti-tuberculosis drugs with clinical and microbiological relevance. Int J Tuberc Lung Dis. 14, 282-288 (2010). 
14. Krüüner, A., Yates, M. D. \& Drobniewski, F. A. Evaluation of MGIT 960-based antimicrobial testing and determination of critical concentrations of first- and second-line antimicrobial drugs with drug-resistant clinical strains of Mycobacterium tuberculosis. J Clin Microbiol. 44, 811-818 (2006).

15. Canetti, G. et al. Advances in techniques of testing mycobacterial drug sensitivity, and the use of sensitivity tests in tuberculosis control programmes. Bull World Health Organ. 41, 21-43 (1969).

16. Peñuelas-Urquides, K. et al. Measuring of Mycobacterium tuberculosis growth. A correlation of the optical measurements with colony forming units. Braz J Microbiol. 44, 287-290 (2013).

17. Parsons, L. M., Somoskovi, A., Urbanczik, R. \& Salfinge, M. Laboratory diagnostic aspects of drug resistant tuberculosis. Front Biosci. 9, 2086-2105 (2004).

18. Wikler, M. A. et al. Methods for Dilution Antimicrobial Susceptibility Tests for bacterial that grow aerobically: Approved Standard (Clinical and Laboratory Standards Institute, Wayne, PA) (2009).

19. Choi, J. I. et al. A rapid antimicrobial susceptibility test based on single-cell morphological analysis. Sci. Transl. Med. 6, 267ra174 (2014).

20. Park, Y. K. et al. Whole-genome sequence of Mycobacterium tuberculosis Korean strain KIT87190. Genome Announc. 2, e01103-14 (2014).

21. Rodwell, T. C. et al. Predicting extensively drug-resistant Mycobacterium tuberculosis phenotypes with genetic mutations. J Clin Microbiol. 52, 781-789 (2014).

22. Jnawali, H. N. et al. Characterization of mutations in multi- and extensive drug resistance among strains of Mycobacterium tuberculosis clinical isolates in Republic Korea. Diagn Microbiol Infect Dis. 76, 187-196 (2013).

23. Powell, M. et al. Development of in vitro susceptibility testing criteria and quality control parameters-Forth Edition: Approved Guideline M23. (Clinical and Laboratory Standards Institute, Wayne, PA) (2016).

24. Suo, J., Chang, C. E., Lin, T. P. \& Heifets, L. B. Minimal inhibitory concentrations of isoniazid, rifampin, ethambutol, and streptomycin against Mycobacterium tuberculosis strains isolated before treatment of patients in Taiwan. Am Rev Respir Dis. 138, 999-1001 (1998).

25. Woodley, C. L. Evaluation of streptomycin and ethambutol concentrations for susceptibility testing of Mycobacterium tuberculosis by radiometric and conventional procedures. J Clin Microbiol. 23, 385-386 (1986).

26. Craig, W. A., Bhavnani, S. M. \& Ambrose, P. G. The inoculum effect: fact or artifact? Diagn Microbiol Infect Dis. 50, 229-230 (2004).

27. Udekwu, K. I., Parrish, N., Ankomah, P., Baquero, F. \& Levin, B. R. Functional relationship between bacterial cell density and the efficacy of antibiotics. J Antimicrob Chemother. 63, 745-757 (2009).

28. Collins, L. A. \& Franzblau, S. G. Microplate alamar blue assay versus BACTEC 460 system for high-throughput screening of compounds against Mycobacterium tuberculosis and Mycobacterium avium. Antimicrob. Agents Chemother. 41, 1004-1009 (1997).

29. Coll, F. et al. Rapid determination of anti-tuberculosis drug resistance from whole-genome sequences. Genome Med. 7, 51 (2015).

30. Chosewood, L. C. et al. CDC/NIH biosafety in microbiological and biomedical laboratories. http://www.cdc.gov/od/ohs/biosfty/ bmbi15/bmb15toc.htm (2007).

31. Heifets, L. B. \& Cangelosi, G. A. Drug susceptibility testing of Mycobacterium tuberculosis: a neglected problem at the turn of the century. Int J Tuberc Dis. 3, 564-581 (1999).

32. Maglio, D., Ong, C., Banevicius, G. Q., Nightingale, C. H. \& Nicolau, D. P. Determination of the in vivo profile of cefepime against extendedspectrum-beta-lactamase-producing Escherichia coli at various inocula. Antimicrob Agents Chemother. 48, 1941-1948 (2004).

33. Engstrom, A., Microcillo, N., Hoffner, S. E. \& Jureen, P. Detection of first- and second-line drug resistance in Mycobacterium tuberculosis clinical isolates by pyrosequencing. J Clin Mirobiol. 50, 2026-2033 (2012).

34. Chakravorty, S. et al. Rapid detection of fluoroquinolone-resistant and heteroresistant Mycobacterium tuberculosis by use of sloppy molecular beacons and dual melting-temperature codes in a real-time PCR assay. J Clin Microbiol. 49, 932-940 (2014).

35. Attori, S., Dunbar, S. \& Clarridge, J. E. Assessment of morphology for rapid presumptive identification of Mycobacterium tuberculosis and Mycobacterium kansasii. J Clin Microbiol. 38, 1426-1429 (2000).

\section{Acknowledgements}

This research was supported by a grant of the Korea Health Technology R\&D Project through the Korea Health Industry Development Institute (KHIDI), funded by the Ministry of Health \& Welfare, Republic of Korea (grant no: HI13C1468 and HI16C1567); Industrial Technology Innovation Program (10050991) funded by the Ministry of Trade, industry \& Energy (MI, Korea).

\section{Author Contributions}

Y.-G.J. (Myongji University), S.K. (Seoul National University, QuantaMatrix Inc.) and H.K., S.R. (Korean Institute of Tuberculosis) contributed to the concept and design of the study and writing the manuscript. Y.-G.J. and H.K. designed experiments and prepared the Figures. Y.-G.J., S.L., E.J., J.C., H.J.K., J.Y. (QuantaMatrix Inc.) and H.K., H.-J.L., H.K., H.J. (Korean Institute of Tuberculosis) contributed to the performance of the experiments and data analysis. E.-G.K. (QuantaMatrix Inc.) contributed to the design and production of the DST chip.

\section{Additional Information}

Supplementary information accompanies this paper at https://doi.org/10.1038/s41598-018-26419-Z.

Competing Interests: S.L., S.K. (QuantaMatrix Inc.), E.J., E.-G.K., H.J.K. and S.K. (QuantaMatrix Inc., Seoul National University.) at the time of manuscript submission, were employed at QuantaMatrix, Inc., which is commercializing the DST technology. S.L., S.K., E.J., E.-G.K., H.J.K., and S.K. (QuantaMatrix Inc.) have equity interest in QuantaMatrix, Inc.

Publisher's note: Springer Nature remains neutral with regard to jurisdictional claims in published maps and institutional affiliations.

Open Access This article is licensed under a Creative Commons Attribution 4.0 International

License, which permits use, sharing, adaptation, distribution and reproduction in any medium or format, as long as you give appropriate credit to the original author(s) and the source, provide a link to the Creative Commons license, and indicate if changes were made. The images or other third party material in this article are included in the article's Creative Commons license, unless indicated otherwise in a credit line to the material. If material is not included in the article's Creative Commons license and your intended use is not permitted by statutory regulation or exceeds the permitted use, you will need to obtain permission directly from the copyright holder. To view a copy of this license, visit http://creativecommons.org/licenses/by/4.0/.

(c) The Author(s) 2018 